to improve delivery of care. However, prediction models used in medical research often fail to accurately predict health outcomes due to methodological limitations. These models particularly perform poorly when predicting narrowly targeted subgroups of patients. We explore the role of latent class regression (LCR) analysis to model the survival of patients with CHF. We seek to show that using LCR improves the modelling of health outcomes as it accounts for unobserved heterogeneity that exists naturally within the patient data.

Methods LCR generally involves identifying hidden latent classes within data and uses patient's demographic characteristics and other covariates to predict class membership and separate regression models for each class. These latent classes may correspond to subgroups of patients with specific characteristics that affect their survival. The rationale is that one class will be more susceptible to deaths compared to another. The United Kingdom Heart Failure Evaluation and Assessment of Risk Trial (UK-HEART) recruited patients with signs and symptoms of CHF between July 2006 and December 2014. A total of 1802 records were available on patient characteristics as well as medications. We used some of these variables to model survival of patients within a latent class framework by estimating a single regression model for both latent classes. We increased complexity of our model by allowing each class to have a separate survival model.

Results We used the area under the receiver operating characteristic (ROC) curve to assess the performance of these two class models. Overall, our novel approach performed better than the traditional one-model-fits-all approach. Our model gave an area under the curve (AUC) of 0.87 while the traditional model yielded an AUC of 0.68 .

Conclusion Ignoring the natural heterogeneity that exists within the patient data affects the accuracy of estimates in prediction models. Researchers can utilise the available data to identify hidden latent classes within the data. Fitting a regression model to each latent class improves the accuracy of the prediction estimates.

\section{P16 STIGMATIZATION OF SMOKERS: THE ROLE OF SCHOOL TOBACCO POLICIES}

PO Robert* ${ }^{*}$ A Grard, N Mélard, V Lorant. Institute of Health and Society (IRSS), UCL, Bruxelles, Belgium

\subsection{6/jech-2018-SSMabstracts. 142}

Background Stigma exists when components of labeling, stereotyping, separation, and discrimination occur together in a power situation. This may apply to smoking, a behavior being increasingly denormalized. However, it is unknown whether School Tobacco Policies (STP) may lead to the stigmatizationof adolescent smokers. Our aims are (1) to measure the magnitude of eachstigmatization's components and (2) to assess how STPs influence them.

Methods Data on 11493 adolescents in 43 schools in 7 European countries were used (SILNE R-survey, 2016). Dependent variable was Stuber's scale to measure stigmatization with four items: most people think less of person who smoke, believe that smoking is for losers, most non-smokers would be reluctant to date someone who smokes and would not hire a smoker to babysit children. Independent variables were: being a weekly smoker, having friends smoking, parental smoking and the score of school tobacco policies (STPs).
We performed multilevel regressions which include interactions between weekly smoking and other independent variables.

Results The most frequent stigmatization components were related to discrimination such as 'not to hire a smoker as a babysitter' (77\%) and not 'dating a smoker' (55\%). Smokers always reported less stigmatization compared to non-smokers. Differences between smokers and non-smokers were more pronounced for 'not to hire a smoker as a babysitter $(62 \%$ vs $\left.80 \%, \mathrm{X}^{2}=182, \mathrm{p}<0,001\right)$ and less so for stereotype: 'most people think less of person who smokes' (Smokers: 45\%; nonsmokers: $\left.52 \%, \mathrm{X}^{2}=15, \mathrm{p}<0,001\right)$. Multilevel regression showed that stigmatization was lower in smokers, increased with none of my friends being smokers, decreased with parental smoking. In addition, smokers felt more stigmatized in schools with higher STPs score.

Conclusion Smoking is associated with a loss of status. STPs increase stigmatization within school while social environment (friends and family) decrease stigmatization. This suggest that social ties may reduce the effect of stigmatization on smoking behavior among adolescents.

\section{P17 ORGANISATIONAL STAKEHOLDERS WHO ENGAGE IN SCOTTISH E-CIGARETTE POLICY DEBATES: A MIXED METHODS APPROACH}

${ }^{1} S$ Hilton*, ${ }^{1} \mathrm{~T}$ Ikegwuonu, ${ }^{2} \mathrm{~K}$ Smith, ${ }^{3} \mathrm{H}$ Weishaar. ${ }^{1} \mathrm{MRC} / \mathrm{CSO}$ Social and Public Health Sciences Unit, University of Glasgow, Glasgow, UK; ${ }^{2}$ Social Policy, School of Social and Political Sciences, University of Edinburgh, Edinburgh, UK; ${ }^{3}$ Public Health, Hertie School of Governance, Berlin, Germany

\subsection{6/jech-2018-SSMabstracts. 143}

Background Electronic cigarettes (e-cigarettes) have become subject to highly contested public and political debates, including the role of commercial stakeholders in development and implementation of e-cigarette policy. There are concerns that e-cigarette debates provide opportunities for commercial stakeholders to demonstrate alignment with public health interests, build reputation, and gain influence over policy processes. While previous research on commercial sector engagement in policymaking has enhanced understanding of its impact on public health, a striking research gap exists regarding commercial actors' engagement in e-cigarette debates.

Setting Taking the Scottish context as a case study, this project aims to increase understanding of commercial stakeholders' engagement in policy debates on e-cigarettes, generate debate on the sector's engagement in e-cigarette policy, and contribute to the development of effective e-cigarette policy. The project is investigating commercial stakeholders' interests in, and positions on, the benefits, harms and regulation of e-cigarettes, the ways in which interests and positions are presented and evidence is framed, and efforts to build collaboration and shape e-cigarette policy.

Methods A mixed-method approach has been applied, combining the use of social network analysis to systematically analyse the relationships between commercial and other policy stakeholders and thematic analysis of documentary and interview data to explore the nature of commercial stakeholders' engagement in policy debates on e-cigarettes in Scotland. Publicly available policy documents and data from semi-structured, in-depth interviews with key stakeholders have been analysed.

Results The analysis focuses on stakeholders' interests in, and positions on, the benefits and harms of e-cigarettes, the ways 